\title{
EARLY STAGE DIGITAL TWINS FOR EARLY STAGE ENGINEERING DESIGN
}

\author{
Jones, David Edward; Snider, Chris; Kent, Lee; Hicks, Ben \\ University of Bristol
}

\begin{abstract}
While extensive modelling - both physical and virtual - is imperative to develop right-first-time products, the parallel use of virtual and physical models gives rise to two interrelated issues: the lack of revision control for physical prototypes; and the need for designers to manually inspect, measure, and interpret modifications to either virtual or physical models, for subsequent update of the other. The Digital Twin paradigm addresses similar problems later in the product life-cycle, and while these digital twins, or the "twinning" process, have shown significant value, there is little work to date on their implementation in the earlier design stages. With large prospective benefits in increased product understanding, performance, and reduced design cycle time and cost, this paper explores the concept of using the Digital Twin in early design, including an introduction to digital twinning, examination of opportunities for and challenges of their implementation, a presentation of the structure of Early Stage Twins, and evaluation via two implementation cases.
\end{abstract}

Keywords: Design engineering, Design informatics, Design management, Early design phases, New product development

\section{Contact:}

Jones, David Edward

University of Bristol

Mechanical Engineering

United Kingdom

david.jones@bristol.ac.uk

Cite this article: Jones, D.E., Snider, C., Kent, L., Hicks, B. (2019) 'Early Stage Digital Twins for Early Stage Engineering Design', in Proceedings of the 22nd International Conference on Engineering Design (ICED19), Delft, The Netherlands, 5-8 August 2019. DOI:10.1017/dsi.2019.262 


\section{INTRODUCTION}

An orchestration of physical and virtual models of varying fidelities, and in differing sequences, is required for the product development process. The choice of these models depends upon the skills of the design team, resources and tools available, purpose of the model, and nature of the design task. In all engineering disciplines a combination of virtual and physical models is necessary to support the progression of the design process, with each model and iteration thereof generating new understanding and knowledge to inform decision-making.

While extensive modelling - both physical and virtual - is imperative to develop right-first-time products, the parallel use of virtual and physical models gives rise to two interrelated issues: the lack of revision control for physical prototypes; and the need for designers to manually inspect, measure, and interpret modifications to either virtual or physical models, for subsequent update of the other. This manual process of revision control for physical models impacts on the cost, quality and time of the design and development process, and makes optimisation of the product development process in terms of the digital-physical tool-chain all but impossible.

The Digital Twin paradigm addresses similar problems later in the product life-cycle, once the product exists as either a virtual representation to be manufactured, or a physical product to be virtualised. Consisting of the physical system, virtual model and the data connections between, the Digital Twin sees a near real-time coupling of the virtual and physical such that they are identical from a macro to a micro level. The twinning process is largely automatic, with, for example, the physical-to-virtual being performed by Internet-of-Things sensors and the virtual-to-physical managed through actuators.

While these digital twins, or the "twinning" process have shown significant value, there is little work to date on their implementation throughout the entire product lifecycle, and in particular the earlier design stages prior to production. Where twinning has for products in-operation allowed near realtime synchronisation and optimisation, the design and development stages remain largely manual and, from a virtual perspective, given lower levels of support. With large prospective benefits in increased product understanding, performance, and reduced design cycle time and cost, there is value in exploration of applying the twinning approach to earlier design stages. It is this exploration that this paper presents, including an introduction to digital twinning, examination of opportunities for and challenges of their implementation, a presentation of the structure of Early Stage Twins, and evaluation via two implementation cases.

\section{DIGITAL TWINS}

The digital twin is a virtual representation of a physical artefact that contains all the information required to fully describe it. This recent concept was devised by Michael Grieves and John Vickers in their white paper on the origins of the Digital Twin (Grieves (2014)), where the concept is attributed to a 2003 lecture on project life-cycle management and the term "Digital Twin" coined by Vickers of NASA.

Within the white paper, Grieves described the Digital Twin of consisting of three parts: the physical artefact, the virtual representation, and the data connections that feed the virtual with real-time data from the physical. These three parts effectively allow for a 'closed loop' approach to the product life-cycle as real-world performance of the as-built can be compared against as-designed.

Grieves outlined the benefits of such an approach as conceptualisation, comparison, and collaboration. Representing information around a virtual representation of the product contextualises that information in the real-world product. Minimising the cognitive load that would otherwise be required to conceptualise that information. Comparison between the virtual and physical product, or as-designed - as-built comparison allows engineers to monitor performance and verify design decisions. Collaboration is enabled by the Digital Twin via multiple user access to the virtual model such that one could, for example, be supporting the physical production line from the other side of the world.

In the time since its initial conception, the Digital Twin has grown to become a field of research in its own right and has seen the realisation of a number of other benefits including:

- $\quad$ Simulation and modelling during design to evaluate design decisions (Tao et al. (2018))

- Data driven design as the performance and usage of existing products can be used to inform and underpin design rational (Tao et al. (2018))

- Performance prediction including health monitoring and optimisation (Glaessgen et al. (2012)) 
- Improved recycling and recondition as the Digital Twin contains the material and assembly instructions required for complete disassembly and repair (Ayani et al. (2018))

The twinning process begins with either a virtual or physical artefact with the process itself the transition between the virtual-to-physical (via realisation methods) or physical-to-virtual (through metrology). Figure 1 depicts this cycle with Table 1 providing the definitions for each stage. The core benefits of twinning are enabled via increased accuracy, increased fidelity, reduced time cost, and reduced workload in these realisation and metrology stages. Reducing the manifestation of these stages via sensing and manufacturing technologies enables the use of high-fidelity simulations to understand in detail the behaviour/performance of systems, and the rapid intervention/optimisation of systems based on this detailed understanding to actively improve their performance.

Table 1. The twinning cycle

\begin{tabular}{|l|l|}
\hline Term & Description \\
\hline Virtual & $\begin{array}{l}\text { A complete virtual description of a physical product that is } \\
\text { accurate to both micro and macro level. }\end{array}$ \\
\hline Realisation & $\begin{array}{l}\text { The means of converting the virtual description of the prod- } \\
\text { uct into the physical counterpart. Achieved through man- } \\
\text { ufacturing, actuators (e.g. electronic motors), and human } \\
\text { interaction (e.g. replacing a faulty component). }\end{array}$ \\
\hline Physical & $\begin{array}{l}\text { The embodied physical and functional descriptions of the } \\
\text { design-in-progress. }\end{array}$ \\
\hline Metrology & $\begin{array}{l}\text { The means of updating the virtual description using } \\
\text { the real-time state of the physical product. Achieved } \\
\text { through Internet-of-Things sensors, engineering measure- } \\
\text { ments (e.g. laser scan), and user feedback for examples. }\end{array}$ \\
\hline
\end{tabular}

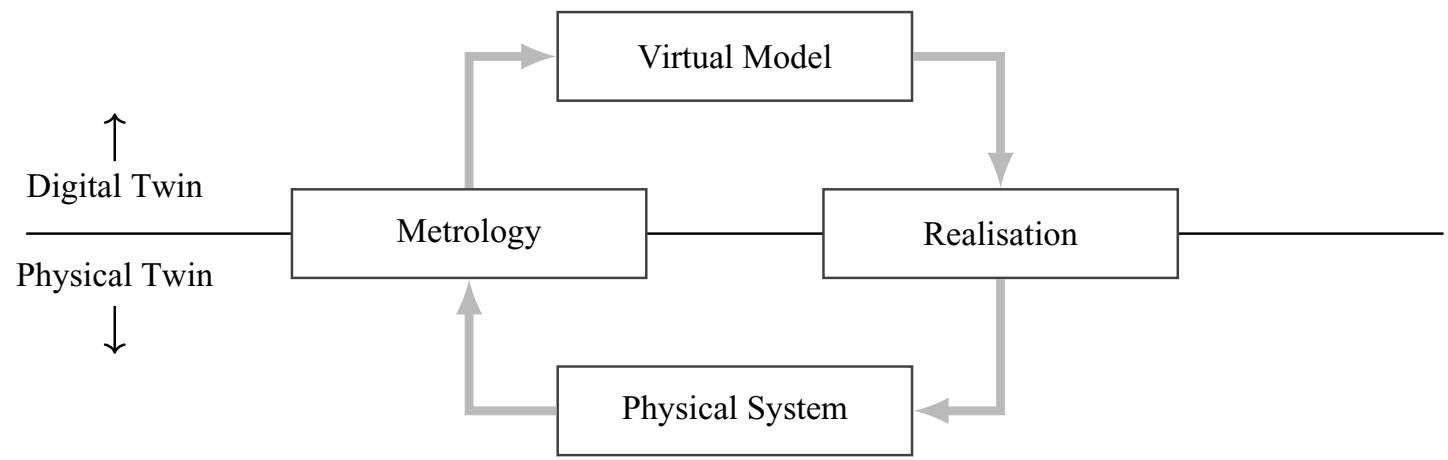

Figure 1. The "twinning cycle"

The twinning cycle begins with a change in the state of either the physical system or virtual model, which is then propagated though to the virtual/physical counterpart. For physical-to-virtual twinning, technology such as Internet-of-Things captures the real-world state of the physical and updates the virtual model accordingly. For the virtual-to-physical twinning, the physical system is changed based on the virtual state using technology such as electrical actuators. A "win" should not be considered the virtual model or physical system alone; it must also include the data connections via realisation and metrology that enable the twinning process. The important factor with digital twinning as depicted in this cycle, is the physical/virtual form and function are largely consistent, with state changes relating to changes in performance and behaviour.

\section{DIGITAL TWINNING IN EARLY STAGE ENGINEERING DESIGN}

Effective engineering design requires a significant evaluative effort at all process stages. Consisting of task clarification, concept, embodiment, and detail stages (Pahl \& Beitz (2014)) prior to production and through-life operation, see Table 2 for descriptions, there is an ever-present need to assess the preliminary design solutions developed against requirements, the problem, and performance and 
behavioural characteristics. Resting prior to production and operation, these design process stages lie prior to lifecycle stages in which the value of twinning has been realised to date.

The quality of an output and extent to which it satisfies requirements is in-part dependent on the evaluative capability of the engineer, and their ability to understand the behaviour, performance, and impact of the solution principles that have been proposed. This is captured in design process models, such as co-evolution of problem-solution spaces, where understanding of the problem and proposed solutions develop by building outputs of evaluation of one into the other (Smulders et al. (2009)), and FBS models (Gero (1990)) where actual behaviour based on solution proposals is evaluated against expected behaviour based on requirements. Effective evaluation is therefore vital.

Table 2. Descriptions of stages of the design process

\begin{tabular}{|l|l|}
\hline Stage & Description \\
\hline Task Clarification & $\begin{array}{l}\text { Generation of a complete description of the problem } \\
\text { to be solved including requirements, via stakeholders, } \\
\text { market analysis, iteration of previous versions, etc. }\end{array}$ \\
\hline Concept Design & $\begin{array}{l}\text { Determination of the primary functions of the solu- } \\
\text { tion, typically via preliminary exploration and testing of } \\
\text { system behaviours. }\end{array}$ \\
\hline $\begin{array}{l}\text { Embodiment } \\
\text { Design }\end{array}$ & $\begin{array}{l}\text { Determination of the detailed behaviour of the solution, } \\
\text { typically through preliminary exploration and testing of } \\
\text { system structure. }\end{array}$ \\
\hline Detail Design & $\begin{array}{l}\text { Determination of detailed system structure, consis- } \\
\text { tent with prior determined behaviours, functions, and } \\
\text { requirements. }\end{array}$ \\
\hline
\end{tabular}

To support effective design, there is a clear need for effective information generation and evaluation of proposed solutions and concepts. These are core areas of benefit for digital twins (see Section 2), where digitisation allows detailed simulation and analyses to be integrated into design, optimisation, and maintenance processes. By applying this paradigm to earlier design process stages, there may then exist scope for increased evaluative capabilities leading to higher quality design outputs. In these earlier stages, the ability to leverage computational analyses is of particular value, mitigating costly rework and ensuring appropriate decisions are made in processes stages that typically dedicate a majority of total project budget.

This section explores the application of twinning to earlier design stages (defined as those within Table 2 and with focus towards pre-embodiment) via potential opportunities, the landscape created of earlier design stages into which twinning must integrate, and challenges in implementation.

\subsection{Opportunities and value of twinning in earlier design stages}

Taking directly from the benefits of digital twins implemented through life, a number of potential opportunities for support exist. These leverage the computational power of the digital world to augment the capabilities of the engineer, while simultaneously supporting their use of physical media.

- Computational Offloading - Typical digital twins utilise detailed simulations to provide highfidelity results and allow fine-grain optimisation (Tao et al. (2018)). As applied in earlier stages, computational analyses may rapidly apply such simulations that determine system performance of design alternatives right from earliest opportunity. This would give engineers high-fidelity understanding of the behaviour of their design alternatives, both reducing their evaluative effort and greatly increasing detail and breadth of understanding.

- Data-Driven Design - Where early stage evaluation is often a manual process dependent on the skill of the engineer, digitisation creates opportunity to leverage the rapidly growing capabilities of data science (e.g. machine learning, big data analysis) for new levels of design understanding.

- Revision Management and Control - Product Data Management (PDM) systems have revolutionised information management and engineering workflow through tight control and structure of digital files and models. By twinning physical to digital, scope exists to extend this control to physical design representations as they are made, allowing changes made and capabilities of each 
to be stored and analysed for future usage. Such information access may greatly streamline future design changes and iterations.

- Visualisation and and Immersive technologies - Digital / physical synchronisation creates new opportunities for visualisation of data and designs. Recent leaps in hardware and software development have revolutionised the way in which people access the digital world, with technologies such as Virtual and Augmented Reality (VR and AR) being identified as dominant future technologies.

- Streamlined Workflow - Engineers may opt to work in the digital or physical medium that best suit tasks at hand, importantly allowing them to rapidly change their active mechanism without time-cost of transferring their idea between digital/physical forms.

\subsection{Landscape for twinning in early design stages}

A core characteristic of earlier design stages is the typicality of higher levels of manual engineer effort. While the capabilities of the digital world have grown in recent years, much early design activity is highly tailored to the specific system or activity at hand and cannot be easily offloaded to computation. For example, while CAD models are virtual, an engineer typically must go through the manual effort of their creation, physical prototypes are often manually manufactured (i.e. card and clay modelling), and simulations must be manually set up for the specific inputs of the current iteration of the design.

Further, a lack of information often exists in earlier stages. This takes many forms, including detail of the problem space and context, user needs and requirements, and performance of proposed solutions.

Coupled with core characteristics of early design and the activities that engineers perform, this creates a landscape with which early stage twins must fit for their benefits to be realised.

- Exploration - As core to finding highly effective, innovative, or creative solutions, the act of exploration of a design space and design alternatives is vital in ensuring that effective solution principles are found. Thus, a need for effective evaluation of many different alternatives exists. Many formalised methods exist, including to characterise performance against specifications (i.e. QFD), and to compare, select, and evaluate solution principles relative to one another (i.e. morphological charts, weighted tables). Here, digital twinning must actively support exploration of alternatives in a flexible manner that does not restrict the solutions that can be considered, and allow a variety of analytics to be applied.

- Analysis - Evaluation of a design solution is often dependent on analysis of its features, behaviour, and structure in comparison to problem descriptions and needs. The eventual success of a solution can be dependent on analysis ranging from highly technical to purely subjective, and the process of determining performance in each area can be challenging. Digital twinning should therefore support multiple forms of analysis, including of technical performance (i.e. FEA, CFD) and against requirements (i.e. cost, complexity), to support engineer and user evaluation.

- Breadth of Design Representations Used - Particularly at earliest stages, designs may be represented in a variety of abstracted forms (e.g. sketches, system architectures, mixed-media prototypes, geometric models) and may be used for a variety of different purposes (Mathias et al. (2018)). Iterative development and detailing of these preliminary design representations leads to the final design output. Where typical digital twinning utilises a fully-operational machine/system as its subject of analysis, digital twinning in early stages must a) operate using abstracted forms of representation that evolve and detail as the design process continues, and b) allow flexibility in the form of representation used to match current process needs.

- Rapid Iteration Cycles - Much exploration in early process stages aligns with the cognitive design processes of the engineer, including rapid probing of many alternatives to identify preferable design directions through lightweight evaluation. In keeping with the twinning paradigm, high synchronisation rates between digital and physical forms must then be maintained to allow these rapid iterative cycles to occur. This is of particular challenge in earlier stages, where the use of inconsistent and incomplete design representations restricts physical to digital synchronisation through a lack of consistent data inputs and infeasibility of using consistent sensing methods.

- Accessibility - Input of major stakeholders is vital to ensure that requirements are sound and the outputs generated solve the specific problem identified (Toor and Ogunlana (2010)). This creates issue throughout early design as many stakeholders are of lower technical expertise, and struggle to effectively articulate needs or evaluate proposals in sufficient detail to steer future work. Early 
stage twins must be accessible to a broad range of stakeholders including technical engineers and non-technical users, allowing them to understand and contribute to the design process.

\subsection{Challenges for digital twinning in early stages}

This landscape and opportunity bring to the fore a number of challenges for the implementation of digital twins in early design that must be solved from a technological and process perspective.

- Scale of Change and Metrology - Where twinning typically considers smaller structural refinements and optimisations, scale of change in early design stages is potentially much larger. From a technological perspective the physical to virtual transition then requires suitable fidelity to capture macro and micro-level changes to the physical model. This has impact on viable technologies, where fidelities on smaller prototypes may be of sub-mm scale. Further, capture requirements may be highly variable and require integration of multiple technology streams, ranging from pure 3D geometry, to part configuration, to specific characteristics of parts and processes (e.g. cost, time).

- Virtual Data Capture and Structure - Huge effort in data-driven systems is dedicated to appropriate data structuring and manipulation. Given the broad representations used in physical and virtual modelling this raises difficulty for early stage twins - the virtual data structures used must be compatible with a broad variety of data inputs. Further, feasible methods of analysis are highly dependent on the data structures present (i.e. textual data, geometric data, networks), and will have varying analytic capabilities and a non-trivial transfer cost from one format to another. Early stage twins must then use a consistent data structure that allows effective analysis and data manipulation, while simultaneously being flexible to a very broad range of data inputs.

- Manufacture - The physical artefacts used in early stage design vary in form and medium, ranging from sketches to physical prototypes. Purpose of these physical models ranges broadly from solely visual representation to proof-of-function or fully working. Under this backdrop, the manufacturing of physical models is challenging and technology dependent. Effective early stage twins must address challenges in manufacture of physical systems of appropriate form, material, and manufacture process for both physical and activity-driven needs (i.e. for physical testing, for reconfigurability, for gathering user feedback).

- Data Visualisation - As the output of computational analyses, much data visualisation in early stage twinning will abide by widely accepted rules for best practice (see Few (2013)). However, given the need for rapid transfer between virtual and physical, there may be need for methods of visualisation that translate into the physical domain. This highly technical challenge suggests the use of cutting-edge technologies such as virtual and augmented realities, or the direct embedding of information into the physical systems created. Coupled with a wide variety of purposes of visualisation dependent on the broad activities of early stage design, early stage twins must consider effective methods of both 2D visualisation and projection of information into the physical domain.

- Process Planning - A broad number of metrology and manufacturing technologies, data structures, virtual analyses, and physical models will be common in early stage twinning. Accordingly, there is a need to consider effective processes, toolchains, and pipelines to maximise speed and efficiency of the twinning cycle and to maintain compatibility with the purposes and activities present in earlier stage design.

Taking these potential benefits and challenges in implementation, this paper continues by proposing and exploring the implementation of early stage twins - a digital twin paradigm for earlier design.

\section{EARLY STAGE TWINS FOR EARLY STAGE ENGINEERING DESIGN}

Building on the Digital Twin concept and the twinning cycle presented in Figure 1, this section now proposes a new twinning cycle for using within early stage design. The key difference this new twinning process, is it must capture the dynamic form and function associated with early stage designs rather than, for example, the condition or performance monitoring associated with later stage design and the rest of the product life-cycle.

\subsection{The twinning cycle in early-stage design}

Given the characteristics of earlier design stages, the form of each stage of the twinning cycle will be distinct to that seen in typical digital twinning implementations. Core focus here lies in the processes 
of synchronisation between digital and physical models considering the dynamic form and functional descriptions as the design changes. Figure 2 shows the new proposed twinning cycle for the early design phase. The key change to the cycle depicted in Figure 1 is the addition of the extra realisation and metrology stages.

As an example, once the state D-Virtual Model is changed, that change is quantified through DMetrology. This change can then be physically realised though P-Realisation, to create an up-to-date P-Physical System. A change in the state of the P-Physical System is again quantified in P-Metrology, the D-Virtual Model then requires updating to mirror this new state. This is achieved in D-Realisation. These additional steps of realisation and metrology quantify change and update form and functional descriptions, be that within the virtual of physical.

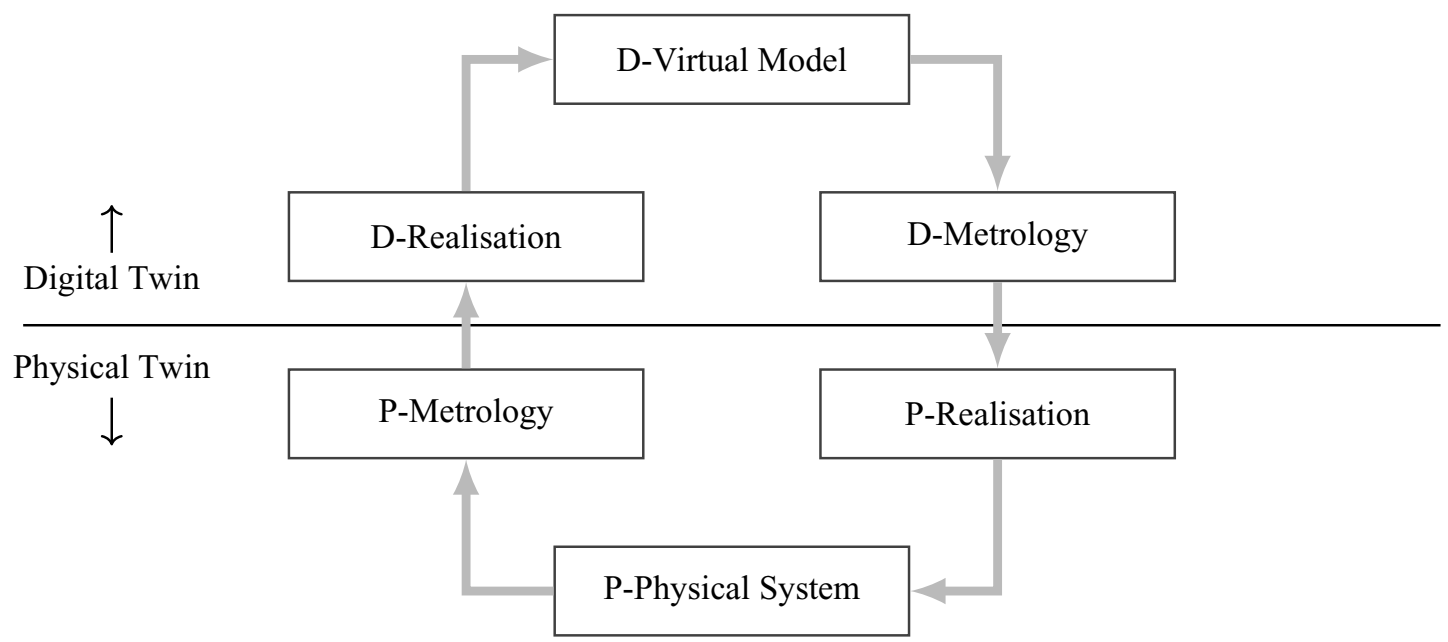

Figure 2. The "twinning cycle" for early stage twins

Table 3. The twinning cycle for early stage twins

\begin{tabular}{|l|l|}
\hline Stage & Description \\
\hline D-Realisation & $\begin{array}{l}\text { The process of updating the virtual description with the } \\
\text { state the physical system. }\end{array}$ \\
\hline $\begin{array}{l}\text { D-Virtual } \\
\text { Model }\end{array}$ & $\begin{array}{l}\text { A complete virtual description of a physical design-in- } \\
\text { progress that is accurate to both micro and macro level. }\end{array}$ \\
\hline D-Metrology & The process of measuring the state of the virtual model. \\
\hline P-Realisation & The process of physically embodying the virtual model. \\
\hline $\begin{array}{l}\text { P-Physical } \\
\text { Model }\end{array}$ & $\begin{array}{l}\text { The embodied physical and functional descriptions of } \\
\text { the design-in-progress. }\end{array}$ \\
\hline P-Metrology & The process of measure the state of the physical system. \\
\hline
\end{tabular}

\subsection{Early stage twins in early stage design}

Very few examples of digital twinning have been implemented in earlier design stages. This section uses two implementations of recognisable early stage twinning systems (see Table 4) to evaluate the potential for the approach.

- City-Blocks (CB) - City-Blocks represents an exploration of the early stage twinning approach and is yet to be fully published. The system comprises a city design demonstrator for non-technical stakeholder engagement with infrastructure design. Users create communities using Lego bricks, where colour represents building type, which are then scanned, analysed, and used to automatically generate a virtual model. The user access the model through virtual reality, evaluates via exploration and automatically calculated metrics of performance, and iterates their design.

- Augmented Reality SandBox (SB) Reed et al. (2014) - From the field of geography and education, the Augmented Reality Sandbox enables users to physically and tangibly design topological 
terrains, which is augmented with a real-time virtual elevation map. Users interact with a physical sandbox that is scanned in real-time to create a contour map of elevation. Using a projector, freshwater eco-systems are overlaid on the sandbox, indicating water flow through the user-created landscape. Users iterate their landscape and evaluate the impact of their actions, in a landscape and eco-system design context.

Table 4. Mapping of implementation cases to the early stage twinning cycle

\begin{tabular}{|l|l|l|}
\hline Stage & City-Blocks (CB) & Sandbox (SB) \\
\hline D - Realise & $\begin{array}{l}\text { Matrix generated from image } \\
\text { data, procedurally generated } \\
\text { CAD model from matrix }\end{array}$ & $\begin{array}{l}\text { Depth-map extracted } \\
\text { from 3D scan data }\end{array}$ \\
\hline D - Virtual Model & $\begin{array}{l}\text { Geometric model and matrix } \\
\text { representation of buildings }\end{array}$ & Contour elevation map \\
\hline D - Metrology & $\begin{array}{l}\text { Analytics of performance run } \\
\text { on matrix, Virtual Reality } \\
\text { exploration of CAD model at } \\
\text { full scale }\end{array}$ & $\begin{array}{l}\text { Contour map projected } \\
\text { over sand (augmented } \\
\text { reality) }\end{array}$ \\
\hline P - Realise & $\begin{array}{l}\text { Users manually iterate Lego } \\
\text { design }\end{array}$ & $\begin{array}{l}\text { Users manually move } \\
\text { sand }\end{array}$ \\
\hline P - Physical System & $\begin{array}{l}\text { Lego interface (bricks on } \\
\text { baseplate) }\end{array}$ & Sand \\
\hline P - Metrology & Webcam-driven scanning & 3D geometric scanning \\
\hline
\end{tabular}

\subsection{Discussion of early-stage twinning}

These implementations, in context of the opportunities, landscape, and challenges described in Section 3 , allow discussion of the feasibility, benefits, and further work requirements of early-stage twinning.

\subsubsection{Opportunities of early-stage twinning}

Both CB and SB show the capability of early stage twinning to enable some of the opportunities proposed in Section 3.1. The digitisation of physical form through largely automatic means in each case creates a virtual model that can be directly used for further analysis (i.e. data-driven design), in one case evaluating performance against predefined metrics and in the other performing complex fluid flow simulation. Running such analyses are typically highly intensive activities that would require significant effort if performed manually, with Early Stage Twins showing feasibility of this effort reduced to near-zero. Further, by digitisation as both a matrix (for the running of analyses) and a geometric CAD model (for virtual reality navigation), CB shows feasibility of generating and using multi-modal virtual models in tandem to support design iterations. Both systems significantly offload the evaluative effort of the engineer to a computational system, which has the multifaceted benefits of reducing engineer workload and reducing design cycle time while simultaneously increasing understanding.

This digitisation process also creates versioning of the physical systems from each design iteration, which while not stored in physical form can be a) directly replicated should need arise, and b) stored as a record of the design process for future analysis and record keeping. This has significant potential benefit for lessons learned and streamlining future design iterations, where engineers can view the previous design versions (and results of evaluation), clarifying the rationale by which designs were formed and reducing the possibility of re-work.

It is of note that in both implementations the act of physically realising changes to the system was actuated by the user - Lego bricks and Sand had to be manually moved. As such, the benefits of streamlining workflow are present physical to virtual transition only, i.e. the time-cost and effort of virtual to physical transformation remains. Further research effort into technologies by which physical realisation could be automated would be highly valuable, if a highly challenging technical task. 


\subsubsection{Compatibility with early stage design}

The combination of technologies used and compatibility with activities performed in each case show the viability of use of early stage twins to support early stage design activities. This evidences the viability of extending the rapidly growing body of work into digital twinning beyond the typical in-operation life-cycle stages into pre-production and design. The use of immersive technologies and highly tangible interfaces combined with near real-time digitisation and visualisation creates a highly accessible environment, where in both cases non-technical users are able to actively evaluate their designs and make intelligent design decisions. This high pace also supports rapid iteration cycles, which in turn enables users/engineers to explore, design, and evaluate solution alternatives efficiently and effectively.

A major difficulty evidenced lies in the highly bespoke nature of every element of the two implementations. Both physical system and virtual model are highly controlled and bounded in order to ensure feasibility of digitisation and ability to augment results of analysis. The method of digitisation and analyses performed are also entirely bespoke for each case. For example, the CB Lego interface must use certain bricks in a confined space for scanning to operate, the geometric model is tied to these constraints, and the analyses run are fixed. As such, while both implementations show capability, they are far from suitably flexible to accommodate the variety of design representations used throughout earlier designs stages. Significant effort would be required to allow any alternative representations to be used, or even to create flexibility in the use of those present beyond tightly predefined constraints. While $\mathrm{CB}$ shows feasibility of the use of multiple forms of virtual model in the same system, bespoke effort must be dedicated to the development and integration of each form, highlighting that the virtual and physical domains both share similar constraints. As such, while early stage twins show effectiveness in early stage design scenarios their use is highly bespoke and with significant set-up cost. There is a need for significant further research and technical development to increase flexibility of each element of the early stage twinning cycle.

\subsubsection{Evaluation of challenges for early stage twins}

As discussed, key challenges in early-stage twinning lie in technological implementation, and in particular in compatibility with different design representations, physical systems, virtual models, metrology methods, and manufacturing. These are substantial challenges that would support the broad implementation of early stage twinning throughout different design scenarios and purposes, and are reflected in each of the those highlighted in Section 3.3. The cases presented show viability of creating an implementation that addresses each each challenge - both are able to detect micro and macro level changes, both demonstrate data structuring and capture, both utilise novel and accessible visualisations. These implementations are however fully tied to specific cases and require study both of how different virtual models / data structures / physical systems may be accommodated, and of which forms are of higher usefulness and value in early-stage design context.

In both implementations the act of physical realisation is performed by the human-in-the-loop, in this case a non-experienced user. For rapid twinning there must be further exploration of the usefulness and viability of automating this process and supporting the user in rapidly creating physical systems. This requires research and development into manufacture methods for early-stage physical systems and prototypes. The benefits are 3-fold: allowing workers to operate in either the physical or virtual domains at will without time-cost of transfer, accurate replication of past design iterations for further study, and decrease of twinning cycle time.

As a result of the lack of flexibility and variety in technologies used, there is no opportunity to explore process planning in these implementations, which must also be a subject for future work.

\section{CONCLUSIONS}

The Digital Twin is seeing a growth in interest as the scientific and commercial communities endeavour to realise the benefits of conceptualisation, comparison, and collaboration. While this work is progressing, there is an obvious gap during the earliest phases of the product life-cycle. This paper addresses this gap by introducing the concept of the Early Stage Twins, a virtual-physical representation of the early stages of the evolving product concept and design.

While significant challenges remain in implementation, two cases are presented that show both viability and value in the use of Early Stage Twins. Key challenges here lie in developing capable technology, 
and enabling early stage twins to flexibly accommodate the myriad design representations that engineers use in early stage design without significant bespoke development.

Much ongoing work exists in this regard suggesting that these challenges, while large, are not insurmountable. For example, the increasing capability of additive manufacture, hybrid prototyping techniques, immersive interaction technologies (virtual and augmented realities), and big data analysis and machine learning are all directly suited to supporting these challenges.

In enabling early-stage twins, significant opportunity to support exploration, evaluation, and engineer decision-making exists, with potential both to increase performance/quality of outputs produced and to reduce design process time.

\section{REFERENCES}

Pahl, G. and Beitz, W. (1984). "Engineering Design: A Systematic Approach", London: Springer.

Smulders, F.E., Dorst, K. and Reymen, I.M. (2009). "Modelling Co-Evolution in Design Practice", In ICED11: 17th International Conference on Engineering Design. Stanford, CA.

Gero, J.S. (1990). "Design Prototypes: A Knowledge Representation Schema for Design”, AI Magazine.

Mathias, D., Hicks, B., Snider, C. and Ranscombe, C. (2018). "Characterising the Affordances and Limitations of Common Prototyping Techniques to Support the Early Stages of Product Development", DESIGN 2018: The 15th International Design Coference. Dubrovnik, Croatia

Toor, S.-R. and Ogunlana, S.O. (2010). "Beyond the 'iron Triangle': Stakeholder Perception of Key Performance Indicators (KPIs) for Large-Scale Public Sector Development Projects", International Journal of Project Management Vol. 28 No. 3

Few, S. (2013). "Information Dashboard Design", Second. Burlingame, CA: Analytics Press.

Grieves, M. (2014). "Digital twin: manufacturing excellence through virtual factory replication", White paper.

Grieves, M. and Vickers, J. (2017). "Digital twin: Mitigating unpredictable, undesirable emergent behavior in complex systems", In Transdisciplinary perspectives on complex systems (pp. 85-113). Springer, Cham.

Tao, F., Cheng, J., Qi, Q., Zhang, M., Zhang, H. and Sui, F. (2018). "Digital twin-driven product design, manufacturing and service with big data", The International Journal of Advanced Manufacturing Technology, Vol. 94 No. 9-12, pp. 3563-3576.

Glaessgen, E. and Stargel, D. (2012, April). "The digital twin paradigm for future NASA and US Air Force vehicles", In 53rd AIAA/ASME/ASCE/AHS/ASC Structures, Structural Dynamics and Materials Conference 20th AIAA/ASME/AHS Adaptive Structures Conference 14th AIAA (p. 1818).

Ayani, M., Ganebäck, M. and Ng, A.H. (2018). "Digital Twin: Applying emulation for machine reconditioning", In 51st CIRP Conference on Manufacturing Systems, Stockholm, May 16-18, 2018 (Vol. 72, pp. 243-248). Elsevier.

Reed, S.E., Kreylos, O., Hsi, S., Kellogg, L.H., Schladow, G., Yikilmaz, M.B., Segale, H., Silverman, J., Yalowitz, S. and Sato, E. (2014, December). "Shaping watersheds exhibit: An interactive, augmented reality sandbox for advancing earth science education", In AGU Fall Meeting Abstracts.

Cheng, J., Chen, W., Tao, F. and Lin, C.L. (2018). "Industrial IoT in 5G environment towards smart manufacturing", Journal of Industrial Information Integration, Vol. 10, pp. 10-19.

Stark, J. (2015). "Product lifecycle management", In Product Lifecycle Management (Volume 1) (pp. 1-29). Springer, Cham.

\section{ACKNOWLEDGMENTS}

The work reported in this paper has been undertaken as part of the twinning of digital-physical models during prototyping project at the University of Bristol, which is funded by the Engineering and Physical Sciences Research Council (EPSRC), grant reference (EP/R032696/1). 